\title{
Intermittent Left Bundle Branch Block - A Challenging Case of Rare Electrocardiogram Phenomenon
}

\author{
Muhammad Ameen \\ Bronx-Lebanon Hospital Center, Internal Medicine, Bronx, NeW York, NY, US
}

DOI: https://doi.org/10.17925/EJAE.2018.04.01.22

W e aim to present this rare phenomenon of intermittent left bundle branch block (LBBB) in a patient with aspergillus endocarditis, as a case for arrhythmia round. Intermittent LBBB is an unusual phenomenon. Though first described over six decades ago, only a few cases have been reported and pathophysiological basis remains unknown.

\section{Keywords}

Intermittent left bundle branch block (LBBB), 2:1 LBBB, Aspergillus bacteremia, Aspergillus endocarditis, aortic valve endocarditis, conduction abnormality in endocarditits

Disclosure: Muhammed Ameen has nothing to declare in relation to this article.

Review Process: Double-blind peer review.

Compliance with Ethics: All procedures were followed in accordance with the responsible committee on human experimentation and with the Helsinki Declaration of 1975 and subsequent revisions, and informed consent was received from the patient involved in this case study.

Authorship: All named authors meet the International Committee of Medical Journal Editors (ICMJE) criteria for authorship of this manuscript, take responsibility for the integrity of the work as a whole, and have given final approval to the version to be published.

open Access: This article is published under the Creative Commons Attribution Noncommercial License which permits any non-commercial use, distribution, adaptation and reproduction provided the original author(s) and source are given appropriate credit. (c) The Author 2018.

Received: 1 April 2017

Accepted: 20 June 2017

Citation: European Journal of Arrhythmia \& Electrophysiology. 2018;4(1):22-23

Corresponding Author: Muhammad Ameen, Bronx-Lebanon Hospital Center, Internal Medicine, 1650 grand concourse, Bronx, New York, NY, 10457, US. E: drmameen151@gmail.com

Support: No funding was received in the publication of this article.

\section{Presentation}

A 73-year-old female with a history of recent bio-prosthetic aortic valve replacement, stroke, hypertension, breast cancer status post-lumpectomy and radiotherapy, and hyperlipidemia was admitted with shortness of breath and tachycardia. Her blood pressure (BP) was 147/65 and her pulse was 105/minute. Respiratory rate was 18 breaths per minute, and temperature was $97.7^{\circ} \mathrm{F}$; oxygen saturation was $100 \%$ on room air. She had regular heart sounds with a $2 / 6$ systolic ejection murmur at the left sternal border. Her pertinent abnormal labs included sodium $122 \mathrm{mEq} / \mathrm{L}$, troponin $0.16 \mathrm{ng} / \mathrm{ml}$ and brain natriuretic peptide $611 \mathrm{pg} / \mathrm{ml}$.

\section{Assessment}

Initial electrocardiogram (ECG) revealed sinus tachycardia at 105 beats per minute with left ventricle hypertrophy (LVH) and repolarisation abnormality (see Figure 1). Her transthoracic echocardiography (TTE) confirmed bio-prosthetic aortic valve dehiscence with aortic regurgitation for which she underwent revision of aortic valve replacement. She had a prolonged hospital course complicated by atrial fibrillation, subdural haematoma and respiratory failure requiring intubation. During this hospitalisation she developed Aspergillus flavus endocarditis of the second bio-prosthetic aortic valve. This was adequately treated with amphotericin $b$ and variconazole for 6 weeks, followed by lifelong suppressive therapy. She was successfully discharged to a skilled nursing facility after a long hospital stay.

\section{Diagnosis}

The patient was re-admitted few weeks later with dyspnoea, oedema and hypoxia. Her repeat TTE showed 50\% dehiscence of the bio-prosthetic aortic valve with vegetation. She developed complete heart block during her third bio-prosthetic aortic valve replacement and epicardial pacing leads were placed. She failed to wean off the ventilator and required tracheostomy and percutaneous endoscopic gastrostomy (PEG) tube placement. Her condition continued to deteriorate. Unfortunately, she did not recover from this severe illness and passed away while in the hospital.

During her last stay in the hospital, her ECG revealed a very complex and interesting phenomenon. Her baseline ECG (see Figure 1) showed sinus rhythm (SR), heart rate $87 / \mathrm{min}$, left ventricular hypertrophy with repolarisation abnormalities and normal PR, QRS and QTC intervals. Her baseline ECG transformed into sinus tachycardia with left bundle branch block (LBBB) pattern (see Figure 2). Atrioventricular (AV) pacemaker was inserted for complete heart block. The following day her ECG pattern showed SR, heart rate of 100/min with alternating LBBB and normal conduction (Figure 3; 2:1 LBBB pattern). She had three different patterns of ECG on the same day. Her fourth ECG shows development of atrial fibrillation with normal conduction. The fifth ECG was back in SR with prolonged QTC and first-degree heart block along with LBBB.

\section{Discussion}

Among all the cardiac valve lesions, aortic valve pathology is the most common to be accompanied by various ECG abnormalities due to its close proximity to the AV node and bundle of His. ECG 
findings related to aortic valve endocarditis include various degrees of heart block, ST elevation, pericarditis like ECG changes and bradyarrythmia or tachyarrhythmia. ${ }^{1}$ Development of cardiac conduction abnormalities in patients with infective endocarditis is one of the most ominous signs and predicts high mortality. Regardless of causative organism, ECG abnormalities are more commonly seen in prosthetic valve endocarditis as compared with native valve and disseminated infections. ${ }^{2}$

Even though Aspergillus can cause diseases in both healthy and immunocompromised populations, invasive Aspergillosis is usually seen in the latter. Our case was unique, as the patient did not have any apparent immunosuppressed state. Disseminated Aspergillosis has been reported to lead to various cardiac conditions, notably AV block, myocarditis, pericarditis, endocarditis, myocardial infarction, aortitis, intracardiac mass and pericardial tamponade. Aspergillus endocarditis is a fatal condition with poor prognosis despite treatment. Blood cultures rarely show Aspergillus. Our patient was among the rare ones who grew Aspergillus on blood specimen.

A close view of our case demonstrated an unusual pattern of normal conduction alternating with LBBB or 2:1 LBBB which is referred to a normal QRS alternating with bundle branch block pattern QRS complex due to blockage of the isolated left bundle branch with constant PR, PP and RR intervals. Friedberg first described in 1949 the interesting phenomenon that was initially named as intermittent bundle branch block. Subsequently, more cases were reported associated with atherosclerotic heart disease. Fenichel et al. in 1977 were the first to document this ECG finding in Staphylococcus aureus endocarditis. ${ }^{3}$

Left bundle branch (LBB) usually gets depolarised either anterograde from sinus impulse (normal conduction pattern) and, if injured, then retrograde from the healthy right bundle branch via trans septal conduction (pattern seen in typical 1:1 LBBB). Anterograde conduction is blocked when sinus cycle length is less than the effective refractory period (ERP) of LBB and next impulse arrives before LBB is recovered from prior conduction. Now impulse is conducted via retrograde conduction to LBB and results in a wide LBBB pattern QRS complex. This is a typical pattern seen in 1:1 LBBB.

The plausible explanation for 2:1 LBBB is that the activation coming from the right bundle branch through the septum can also be blocked retrogradely over the left bundle branch when the sinus rate is fast enough for the activation to fall in the retrograde refractory period of the left bundle branch. This results in anterograde, as well as retrograde block in the left bundle branch and thus does not allow the resetting of the refractory periods (no linking phenomenon). Thus, the next sinus activation will find a non-refractory left bundle branch and LBBB will disappear (see Figure 3). We also observe 1:1 LBBB for slightly higher heart rates, which cannot be explained by the persistence of lack of retrograde conduction over the left bundle branch (even rather favoured by the higher heart rate) since this obligatorily implies a 2:1 LBBB pattern. Conversely, this is the decrease in His-Purkinje tissues refractory periods during elevated heart rates which can explain an enhancement of retrograde conduction and thus a new linking phenomenon. ${ }^{4}$
Figure 1: Baseline electrocardiogram - sinus rhythm, with left ventricular hypertrophy and repolarisation abnormalities

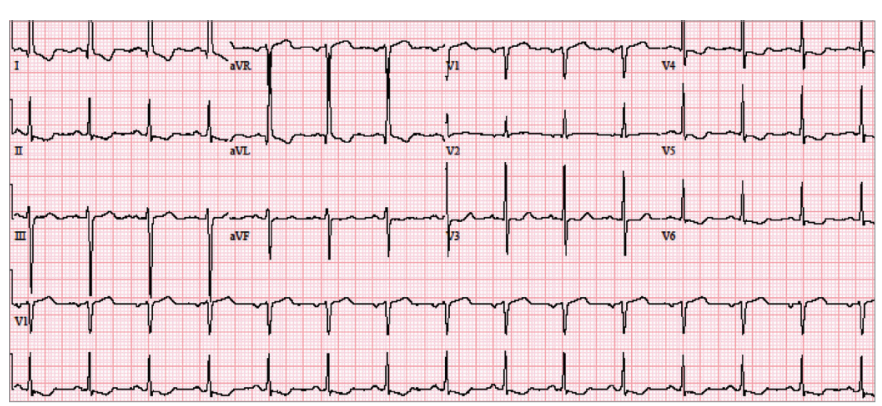

Figure 2: Sinus tachycardia with left bundle branch block

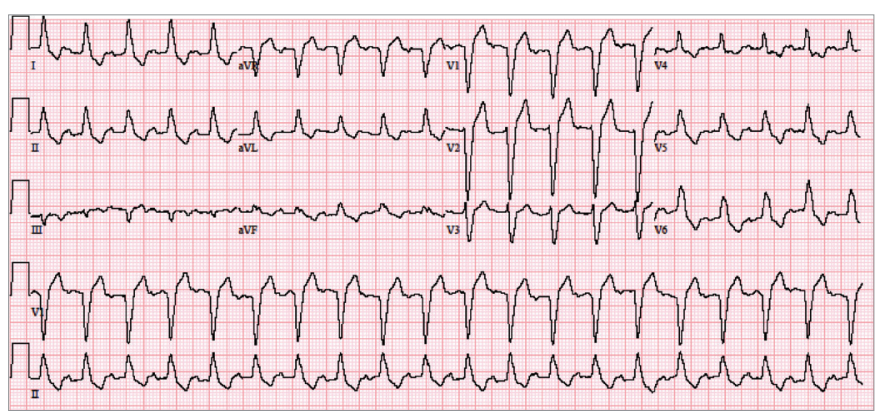

Figure 3: Regular sinus rhythm with narrow QRS complex of $<0.8$ seconds, alternating with wide left bundle branch block pattern QRS complex of 0.14 seconds; PR interval is constant

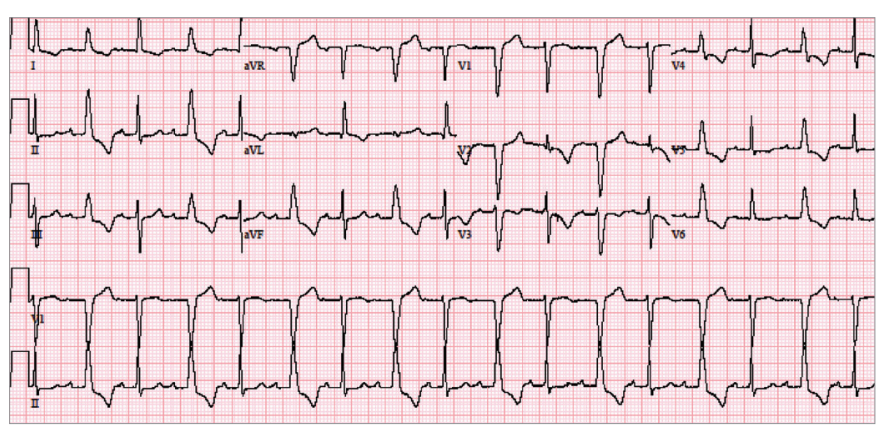

First degree heart block can be easily explained with endocarditisrelated vegetation and abscess formation around the AV node and it has been well documented in the literature. Appearance and disappearance of atrial fibrillation was most likely a cardiac ischaemia induced transient phenomenon. $]$

\footnotetext{
Berk WA, Electrocardiographic findings in infective endocarditis. J Emerg Med. 1988;6:129-32.

2. Meine TJ, Nettles RE, Anderson DJ, et al. Cardiac conduction abnormalities in endocarditis defined by the Duke criteria. Am Heart J. 2001;142:280-5.

3. Fenichel NM, Jimenez FA, Polachek AA, 2:1 left bundle branch block in acute bacterial endocarditis with septal abscess. J Electrocardiol. 1977;10:287-90.

Vereckei A, Tenczer J, Intermittent left bundle branch block: what is the mechanism? J Cardiovasc Electrophysiol. 2003;14:1010-2.
} 